\title{
A METHOD FOR THE CONSTRUCTION OF REFLECTION LAWS FOR A PARABOLIC EQUATION( $\left.{ }^{1}\right)$
}

\author{
BY \\ C. DENSON HILL
}

In this paper we consider the parabolic equation

$$
u_{\tau}=a u_{\xi \xi}+2 b u_{\xi \eta}+c u_{n \eta}+d u_{\xi}+e u_{\eta}+f u
$$

with $b^{2}-a c<0$ whose coefficients are holomorphic functions of $\xi, \eta$ and $\tau$. We present a general method for the construction of explicit reflection formulae (analogous to the classical Schwarz reflection principle for harmonic functions) for solutions of (1.1) which vanish along a noncharacteristic analytic surface.

These formulae (cf. (8.3) and (8.7)) have a domain of dependence consisting, in general, of a one-dimensional curve extending from the reflecting surface to a specific image point. In special cases, however, the domain of dependence may degenerate to just the image point.

An interesting aspect of our technique is the use of fundamental solutions which have singularities not only along the real characteristic $\tau=$ const. $=t$ associated with (1.1) but also on certain complex characteristics as well.

The simplest case of (1.1) is the heat equation

$$
u_{\imath}=u_{\xi \xi}+u_{\eta \eta}
$$

In that case the fundamental solution we use is (cf. (9.15)) the function

$$
i S(x, y, t ; \xi, \eta, \tau) \equiv \frac{1}{(\tau-t)} \exp \left\{\frac{-r^{2}}{4(\tau-t)}\right\} \log \frac{1}{r}+\frac{4}{(\tau-t)} \sum_{j=1}^{\infty} \frac{\gamma_{j}}{j !}\left\{\frac{-r^{2}}{4(\tau-t)}\right\}^{\prime}
$$

Here $r=\left[(x-\xi)^{2}+(y-\eta)^{2}\right]^{1 / 2}$ and $\gamma_{j}=1+1 / 2+\cdots+1 / j$. Observe that, aside from a normalizing factor, the fundamental solution usually associated with the heat equation appears in (1.3) as the coefficient of the logarithmic term. Similarly, the coefficient of $(\tau-t)^{-1}$ in the Laurent expansion of $i S$ is $\log (1 / r)$ which is the fundamental solution of Laplace's equation $u_{\xi \xi}+u_{\eta \eta}=0$. Finally, the coefficient of $(\tau-t)^{-1} \log (1 / r)$ is the constant 1 and that is the Riemann function for the wave equation $u_{v v}-u_{\mu u}=0$ obtained from Laplace's equation by means of the complex substitution $\mu=\operatorname{Im}\{\xi\}, \nu=\operatorname{Re}\{\eta\}$.

The reflection problem has been rather thoroughly investigated for equations in two independent variables. In particular, for linear or nonlinear elliptic equations

Received by the editors May 3, 1967.

(') Present address: Stanford University, Stanford, California. 
of the second order in two variables, Lewy [10] has developed an elaborate theory of reflection across analytic arcs on which analytic boundary conditions are imposed. A principal result of Lewy's theory is that the general linear boundary condition and the boundary condition $u=0$ do not differ with respect to the domain into which they permit extension.

The situation is quite different in higher dimensions. A case in point is the extension of harmonic functions

$$
u_{x_{1} x_{1}}+u_{x_{2} x_{2}}+u_{x_{3} x_{3}}=0
$$

in three variables across a plane boundary on which the linear boundary condition

$$
u_{x_{3}}=A\left(x_{1}, x_{2}\right) u \text { at } x_{3}=0
$$

is imposed. Filippenko [2] established that when $A\left(x_{1}, x_{2}\right)$ is a polynomial in $x_{1}$ and $x_{2}$, the solutions of (1.4), (1.5) have a global continuation property with respect to mirror image domains analogous to the familiar one derived from the classical Schwarz reflection principle for harmonic functions. Recently Lewy [11] gave an example which shows that the same global continuation property does not hold for general analytic $A\left(x_{1}, x_{2}\right)$.

The earlier analysis of Garabedian [3] suggests that Filippenko's result is very special and that Lewy's example is typical. Specifically, Garabedian's work indicates that for a general situation analogous to (1.4), (1.5) the domain of dependence associated with a point on one side of the plane $x_{3}=0$ is a whole threedimensional ball on the other side. Hence, in general, extension into the full mirror image of a domain abutting the plane $x_{3}=0$ should not be expected.

In special cases such as Filippenko's however, some kind of degeneracy may be present which causes the domain of dependence to collapse onto a lower dimensional subset, allowing continuation into a larger region than that afforded in general. Such a phenomenon might be regarded as a type of Huygens' principle for reflection analogous to a Huygens' principle for the Cauchy problem wherein the domain of dependence may degenerate to a lower dimensional subset and thus allow the solution to be extended into a larger region than usual.

In fact virtually all the classic reflection laws [1], [8], [12], [13] were found for situations involving a great deal of symmetry where just such a Huygens' principle is present. In the case of higher order equations with constant coefficients and a plane reflecting boundary, John [9] has found certain necessary conditions on the form of the operator in order that the reflection law be of simple form.

The results of this paper show that in the case of the parabolic equation (1.1), even though it involves three independent variables, the reflection law has a domain of dependence of the same dimension (one) as in Lewy's theory [10] for an elliptic equation in two variables rather than of higher dimension as in Garabedian [3]. Hence it is reasonable to expect that for (1.1) the general linear boundary condition and the boundary condition $u=0$ are also "coextensive". 
2. General remarks on reflection. Let there be given a solution $u$ of a linear partial differential equation which satisfies a linear boundary condition along some noncharacteristic hypersurface contained inside its domain of definition. The generalization of the Schwarz reflection principle, should (if it exists) express the value $u(P)$ of the solution at a point $P$ on one side of the reflecting surface as a linear functional of $u$ and its derivatives involving data strictly on the opposite side of the surface. It is immediately apparent that there is no unique such reflection functional. The problem is to find a reflection functional that is, in some undefined sense, as simple as possible.

A quite general approach to this problem was sketched by Garabedian [3] for the case of an elliptic equation of the second order in $n$ variables with holomorphic coefficients. Garabedian's method is a most natural extension of Hadamard's classic analysis [6]. Two basic ingredients go into it: The first is a fundamental solution designed to solve the Cauchy problem. The second is a reflected fundamental solution tailor-made to fit the particular reflection problem at hand. The reflected fundamental solution plays a role in reflection problems analogous to that played by Hadamard's fundamental solution in the Cauchy problem.

The general strategy can be outlined as follows: First a bilinear functional is built out of Stokes' theorem that is independent of path in the sense that its support can be deformed without changing its value. The fundamental solution is then inserted into the bilinear functional, making it into a path-independent linear functional representing $u(P)$ in terms of its Cauchy data in the complex domain. Next the support of that linear functional is deformed until it rests on the analytic extension of the reflecting surface. The result is an explicit representation of the solution to Cauchy's problem as a linear functional of its data there. Now the prescribed boundary condition imposes a linear relation among the various derivatives featuring in the Cauchy data on the reflecting surface. The idea is then, by taking that relation into account, to construct a reflected fundamental solution that can also be used in the bilinear functional to represent $u(P)$ as a path-independent linear functional, but such that the support of the new linear functional can be deformed away from the reflecting surface back toward the real domain on the side opposite to $P$. Ultimately, perhaps with the aid of further Cauchy problems, $u(P)$ is expressed in the desired form as a linear functional involving data in the real domain on the other side of the surface.

According to the point of view outlined above, the geometrical structure of a reflection law (or of the set of obtainable reflection laws) depends on the relationship in the complex domain between the complex characteristics of the equation under consideration and the analytic extension of the reflecting surface. That relationship is trivial for an elliptic or parabolic equation in one space variable [7]. In the case of two space variables conformal mapping is available and the general case for an equation of the second order can be reduced to the situation in which the reflecting surface is a plane and the principal part of the operator is the 
Laplacian. For more than two space variables such a reduction does not exist in general. If the principal part of the operator has constant coefficients and the reflecting surface is planar to begin with, such a reduction can be effected by a linear transformation. In particular, the addition of lower order terms to the Laplacian and subsequent reflection in a plane does not pose any serious difficulty. A basically more difficult problem in the case of more than two space variables occurs when either the reflecting surface is not a plane or the principal part of the operator contains variable coefficients.

3. Reduction to standard form. Without loss of generality we may consider the equation

$$
\mathscr{L}[u] \equiv u_{x x}+u_{y y}+a u_{x}+b u_{y}+c u-d u_{t}=0 \equiv L[u]-d u_{t}
$$

having holomorphic coefficients $a, b, c, d$ and the boundary condition

$$
u(x, 0, t)=0 \text {. }
$$

For equation (1.1) can be put into the form (3.1) by means of a nonsingular analytic change of variables and division through by a nonvanishing factor. For each $\tau$ one simply maps the two-dimensional Riemannian manifold with metric defined by the principal part of the original operator onto a plane, taking care to choose the resulting one-parameter family of conformal mappings to bè analytic in $t=\tau$. The principal part of the new operator thus obtained consists of the Laplacian multiplied by a nonvanishing factor that can be divided out.

In the procedure described above, any noncharacteristic analytic surface $\phi(\xi, \eta, \tau)=0$ along which $u$ vanishes gets mapped into some other noncharacteristic analytic surface $\Phi(x, y, t)=0$. Then by means of a second one-parameter family of conformal mappings the surface $\Phi=0$ can be mapped onto the plane $y=0$. The form of equation (3.1) is altered in the process only by multiplication of its principal part by another nonvanishing factor that can be divided out.

4. The fundamental solution. In addition to (3.1) we shall also consider the adjoint equation

$$
\mathscr{M}[v] \equiv v_{x x}+v_{y y}-(a v)_{x}-(b v)_{y}+c v+(d v)_{t}=0 \equiv M[v]+(d v)_{t} .
$$

We define a function $S$ of the form

$$
S(x, y, t ; \xi, \eta, \tau) \equiv A(x, y, t ; \xi, \eta, \tau) \log (1 / r)+B(x, y, t ; \xi, \eta, \tau),
$$

with $r=\left[(x-\xi)^{2}+(y-\eta)^{2}\right]^{1 / 2}$, to be a fundamental solution of $(3.1)$ if it satisfies the following requirements:

$1^{\circ} S$ should satisfy $\mathscr{M}[S]=0$ as a function of $(x, y, t)$ and be analytic in its arguments except at $t=\tau$ or $r=0$ where $S$ has a singularity.

$2^{\circ}$ At the parameter point $x=\xi, y=\eta$ we require $A=i /(t-\tau)$.

$3^{\circ}$ The functions $A$ and $B$ should be regular in $x$ and $y$ at $r=0$, but they have an essential singularity in $t$ at $t=\tau$. 
Note that the function $S$ given as an example in (1.3) does satisfy the above conditions.

5. The reflected fundamental solution. Corresponding to any fundamental solution $S$ of (3.1) we define a reflected fundamental solution $S^{*}$ (with respect to $y=0)$ as follows: It has the form

(5.1) $S^{*}(x, y, t ; \xi, \eta, \tau) \equiv A^{*}(x, y, t ; \xi, \eta, \tau) \log \left(1 / r^{*}\right)+B^{*}(x, y, t ; \xi, \eta, \tau)$,

with $r^{*}=\left[(x-\xi)^{2}+(y+\eta)^{2}\right]^{1 / 2}$, and satisfies the requirements:

$1^{\circ} \mathscr{M}\left[S^{*}\right]=0$ except at $t=\tau$ or $r^{*}=0$.

$2^{\circ} A^{*}(x, 0, t ; \xi, \eta, \tau) \equiv A(x, 0, t ; \xi, \eta, \tau)$.

$3^{\circ} A^{*}$ and $B^{*}$ should be regular in $x$ and $y$ at $r^{*}=0$, but have an essential singularity in $t$ at $t=\tau$.

A reflected fundamental solution for the heat equation (1.2), for example, is the function $S^{*}$ obtained from $S$ by replacing $r$ by $r^{*}$ everywhere in (1.3).

We postpone the actual construction of the functions $S$ and $S^{*}$ for the general equation (3.1) until $\$ 9$ and 10.

6. The bilinear functional. We define

$$
H[u, v] \equiv\left\{\left(v u_{x}-u v_{x}+a u v\right) d y d t-\left(v u_{y}-u v_{y}+b u v\right) d x d t-(d u v) d x d y\right\}
$$

and assume $u$ and $v$ to be holomorphic. Since $\bar{\partial} H[u, v]=0$ we obtain

$$
d H[u, v]=\{v \mathscr{L}[u]-u \mathscr{M}[v]\} d x d y d t .
$$

Hence by Stokes' theorem

$$
\int_{D}\{v \mathscr{L}[u]-u \mathscr{M}[v]\} d x d y d t=\int_{\partial D} H[u, v] .
$$

Here $D$ may be taken to be any three-dimensional chain contained in the sixdimensional region of analyticity and the cycle $\partial D$ is its two-dimensional boundary. It follows from (6.2) that the bilinear functional

$$
\int_{\partial D} H[u, v]
$$

is independent of path in the following sense: Its value is the same for any two cycles $\partial D^{\prime}$ and $\partial D^{\prime \prime}$ which are homologous in a region where $u$ and $v$ are analytic solutions of $\mathscr{L}[u]=\mathscr{M}[v]=0$.

In the applications to follow the 2-cycle $\partial D$ in (6.3) will usually be the product $\partial D=\partial R \times \gamma$ of a 1-cycle $\partial R$ in the complex $(x, y)$-space and a 1-cycle $\gamma$ in the complex $t$-plane.

7. The Cauchy functional. Let $u$ be a holomorphic solution of $\mathscr{L}[u]=0$ in a neighborhood of the point $(\xi, \eta, \tau)$.

In place of $v$ we insert the fundamental solution $S$ of $\$ 4$ into the bilinear functional (6.3). We choose the cycle in (6.3) to be the product $\partial R \times \gamma$ of a circle 
$\partial R: r=i \varepsilon, \varepsilon>0$ lying in the plane $x-\xi=$ pure imaginary, $y-\eta=$ pure imaginary, and of a circle $\gamma:|t-\tau|=\delta, \delta>0$ lying in the complex $t$-plane. Note that $\partial R \times \gamma$ avoids the singularities of $S$. If $\partial D$ is any other cycle homologous to $\partial R \times \gamma$ in a region where $\mathscr{L}[u]=0$, we have

$$
\int_{\partial R \times y} H[u, S]=\int_{\partial D} H[u, S] .
$$

Now we compute the residue in (7.1) as the torus $\partial R \times \gamma$ shrinks down to the point $(\xi, \eta, \tau)$. On $\partial R$ we have

$$
\begin{array}{ll}
x-\xi=i \varepsilon \cos \theta, & d x=-i \varepsilon \sin \theta d \theta, \\
y-\eta=i \varepsilon \sin \theta, & d y=i \varepsilon \cos \theta d \theta .
\end{array}
$$

Hence $d x d y=0$ there and

$$
\begin{aligned}
H[u, S] & =\left(S u_{x}-u S_{x}+a u S\right) d y d t-\left(S u_{y}-u S_{y}+b u S\right) d x d t \\
& =\left[S u_{\varepsilon}-u S_{\varepsilon}+i(a \cos \theta+b \sin \theta) u S\right] \varepsilon d \theta d t
\end{aligned}
$$

Because of the form (4.2) of the fundamental solution we have

$$
\lim _{\varepsilon \rightarrow 0} \varepsilon S=0, \quad \lim _{\varepsilon \rightarrow 0} \varepsilon S_{\varepsilon}=\frac{-i}{t-\tau}
$$

Therefore

$$
\begin{aligned}
\int_{\partial R \times \gamma} H[u, S] & =\lim _{\delta \rightarrow 0} \oint_{y} \lim _{\varepsilon \rightarrow 0} \int_{\partial R} H[u, S] . \\
& =\lim _{\delta \rightarrow 0} \oint_{y} 2 \pi i \frac{u(\xi, \eta, t)}{t-\tau} d t \\
& =(2 \pi i)^{2} u(\xi, \eta, \tau) .
\end{aligned}
$$

Thus from (7.1) we obtain

$$
u(\xi, \eta, \tau)=\frac{1}{(2 \pi i)^{2}} \int_{\partial D} H[u, S]
$$

Equation (7.2) represents the solution $u$ of $\mathscr{L}[u]=0$ as a linear functional of its Cauchy data on the two-dimensional cycle $\partial D$. The representation (7.2) is valid, in particular, for any $\partial D$ that can be continuously deformed into the torus $\partial R \times \gamma$ without crossing the singularities of $S$.

If in the process of deforming $\partial D$ we keep $|t-\tau|>0$, then only the remaining singularity where $r=0$ must be avoided. Since $(x-\xi)^{2}+(y-\eta)^{2}=[(x-\xi)+i(y-\eta)]$ $\times[(x-\xi)-i(y-\eta)]$, the two-dimensional manifold along which $r=0$ consists of those points $(x, y)$ such that $|x-\xi|=|y-\eta|$ and $\arg (x-\xi)=\arg (y-\eta) \pm \pi / 2$. Hence any deformation of $\partial D$ is allowed that keeps $|x-\xi| \neq|y-\eta|$ when $(x-\xi)$ and $(y-\eta)$ are orthogonal. 


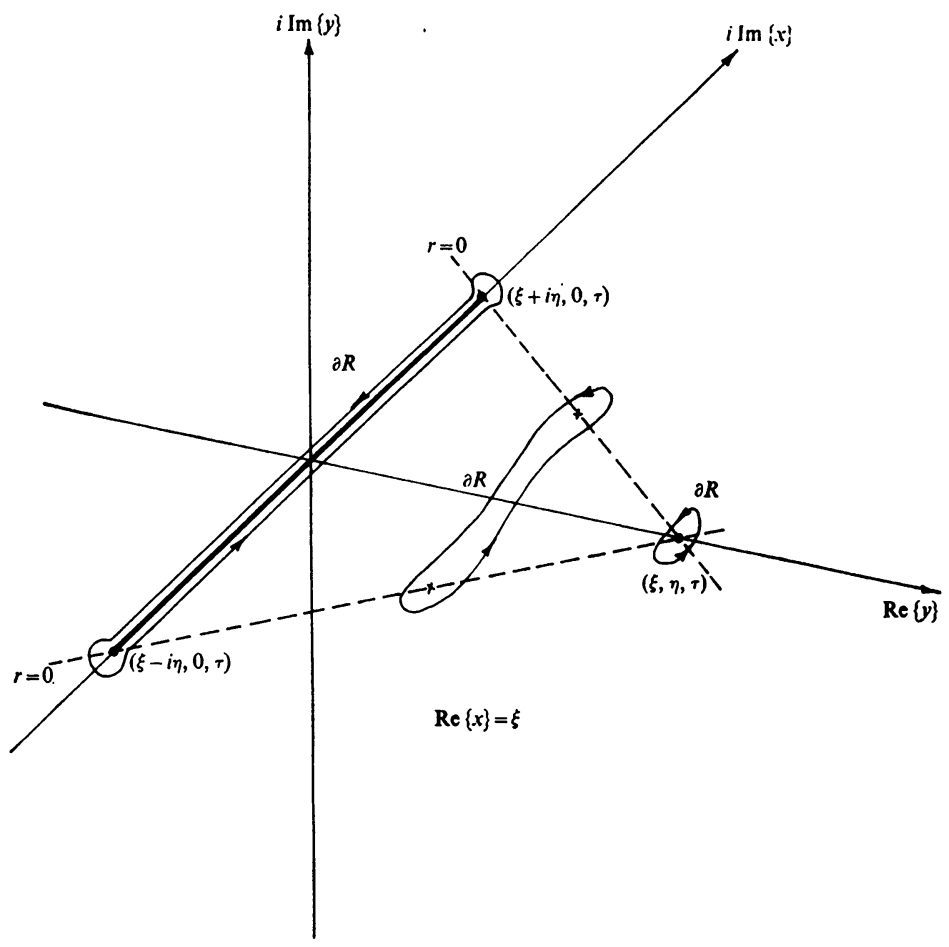

Let $(\xi, \eta, \tau)$ be a point in the real domain with $\eta>0$. Then the intersection of $r=0$ with the plane $y=0, t=\tau$ consists of the two points $(\xi \pm i \eta, 0, \tau)$ which bound the segment $p$

$$
p:\{(x, y, t)|y=0, t=\tau, \operatorname{Re}\{x-\xi\}=0,| \operatorname{Im}\{x-\xi\} \mid \leqq \eta\} .
$$

Now the object is to deform the path $\partial D$ in (7.2) until it forms a torus about $p$ and then to collapse it down onto $p$ obtaining a representation

$$
u(\xi, \eta, \tau)=\lim _{\partial D \rightarrow p} \frac{1}{(2 \pi i)^{2}} \int_{\partial D} H[u, S]
$$

for $u(\xi, \eta, \tau)$ as a residue. Equation (7.3) represents the solution $u$ of $\mathscr{L}[u]=0$ in terms of its Cauchy data on the one-dimensional curve $p$.

The deformation is best visualized as follows: $\partial D=\partial R \times \gamma$ is the product of our circle $\gamma$ and a 1-cycle $\partial R$ lying in the plane $\operatorname{Re}\{y-\eta\}=\lambda, x-\xi=$ pure imaginary, with $-\eta \leqq \lambda \leqq 0$. We hold $\gamma$ fixed and vary $\lambda$ from 0 to $-\eta$, stretching $\partial R$ in the process so as to avoid the singularity at $(x-\xi)= \pm i(y-\eta)= \pm i \lambda$. Then, with $\partial R$ lying in the plane $\operatorname{Re}\{y\}=0$, we collapse $\partial R$ down around the slit

$$
\{(x, y)|y=0, \operatorname{Re}\{x-\xi\}=0,| \operatorname{Im}\{x\} \mid \leqq \eta\} .
$$

The result (7.5) is a certain integral along (7.4) plus residues about each of the endpoints $(\xi \pm i \eta, 0)$. 
The term $B$ in the fundamental solution drops out completely in the process described above, since it is single-valued and regular at $r=0$. Due to our choice of $\partial D$ there is also no contribution from the $d x d y$ term in $H[u, S]$. The only terms in $H[u, S]$ that can contribute to the residues at the points $(\xi \pm i \eta, 0)$ are the ones involving derivatives of $\log (1 / r)$. They yield

$$
\left\{\frac{1}{2 i} A(\xi-i \eta, 0, t ; \xi, \eta, \tau) u(\xi-i \eta, 0, t)+\frac{1}{2 i} A(\xi+i \eta, 0, t ; \xi, \eta, \tau) u(\xi+i \eta, 0, t)\right\} d t .
$$

There is a net contribution along (7.4) only because the logarithm in $S$ is multiplevalued. It is simply $2 \pi i$ (the increment in the logarithm) times the integral along (7.4) of the coefficient of $\log r^{2}$ in $H[u, S]$. Thus the final result is

$$
\begin{aligned}
\frac{1}{2 \pi i} \int_{\partial R} H[u, S]= & \underset{\partial R \rightarrow \text { silt }}{\operatorname{Res}} H[u, S] \\
= & \left\{\frac{1}{2 i} A(\xi-i \eta, 0, t ; \xi, \eta, \tau) u(\xi-i \eta, 0, t)\right. \\
& +\frac{1}{2 i} A(\xi+i \eta, 0, t ; \xi, \eta, \tau) u(\xi+i \eta, 0, t) \\
& \left.+\frac{1}{2 i} \int_{-\eta}^{\eta}\left[A(\xi+i \sigma, 0, t ; \xi, \eta, \tau) u_{y}(\xi+i \sigma, 0, t)-\left(A_{y}-b A\right) u\right] d \sigma\right\} d t \\
\equiv & I[u, A] d t .
\end{aligned}
$$

Hence from (7.2) we have

$$
\begin{aligned}
u(\xi, \eta, \tau) & =\frac{1}{2 \pi i} \oint_{\gamma} \frac{1}{2 \pi i} \int_{\partial R} H[u, S] \\
& =\frac{1}{2 \pi i} \oint_{\gamma} \underset{\partial R \rightarrow \text { sult }}{\operatorname{Res}} H[u, S] \\
& =\frac{1}{2 \pi i} \oint_{\gamma} I[u, A] d t .
\end{aligned}
$$

As a final step we may also compute the residue as $\gamma$ shrinks to a point. Since $A$ has an essential singularity in $t$ at $t=\tau$, the result of that calculation will involve an infinite series. The Laurent expansion of $A$ has the form (cf. \$9)

Since

$$
A=\sum_{j=0}^{\infty} A_{j} j !(t-\tau)^{-j-1}
$$

we obtain

$$
I[u, A]=\sum_{j=0}^{\infty} I\left[u, A_{j}\right] j !(t-\tau)^{-j-1},
$$

$$
\left.u(\xi, \eta, \tau)=\sum_{j=0}^{\infty} \frac{\partial^{j}}{\partial \tau^{j}} I[u, A\}\right]
$$


8. The reflection functional. Let $u$ be a holomorphic solution of $\mathscr{L}[u]=0$ in a neighborhood of some portion of the plane $y=0$ and let $u$ satisfy the boundary condition (3.2). Then the representation (7.5) assumes the simpler form

$$
u(\xi, \eta, \tau)=\frac{1}{2 \pi i} \oint_{\nu}\left\{\frac{1}{2 i} \int_{-\eta}^{\eta} A(\xi+i \sigma, 0, t ; \xi, \eta, \tau) u_{y}(\xi+i \sigma, 0, t) d \sigma\right\} d t
$$

Now we insert the reflected fundamental solution $S^{*}$ into the bilinear functional (6.3) and perform exactly the same residue computation that lead to (7.5). As before the term $B^{*}$ drops out completely. Due to the boundary condition (3.2) all the terms involving $u$ also drop out. Moreover the increments in $\log r^{2}$ and $\log r^{* 2}$ around the points $(\xi \pm i \eta, 0)$ have opposite signs. Thus everything is just the same as before except for a change of sign. Hence we obtain

$$
\begin{aligned}
\frac{1}{(2 \pi i)^{2}} \int_{\partial D^{*}} H\left[u, S^{*}\right] & =\frac{1}{2 \pi i} \oint_{\gamma} \frac{1}{2 \pi i} \int_{\partial R} H\left[u, S^{*}\right] \\
& =\frac{1}{2 \pi i} \oint_{\gamma} \underset{\partial R \rightarrow B l 1 t}{\operatorname{Res}} H\left[u, S^{*}\right] \\
& =\frac{1}{2 \pi i} \oint_{\gamma}\left\{-\frac{1}{2 i} \int_{-\eta}^{\eta} A^{*}(\xi+i \sigma, 0, t ; \xi, \eta, \tau) u_{y}(\xi+i \sigma, 0, t) d \sigma\right\} d t .
\end{aligned}
$$

Since $A=A^{*}$ at $y=0$ this means we actually have

$$
u(\xi, \eta, \tau)=-\frac{1}{(2 \pi i)^{2}} \int_{\partial D^{*}} H\left[u, S^{*}\right]
$$

The representation (8.2) of $u(\xi, \eta, \tau)$ in terms of the reflected fundamental solution $S^{*}$ has an advantage over (7.2) in that the singularities of $S^{*}$ along $r^{*}=0$ bend back from the points $(\xi \pm i \eta, 0)$ and intersect the real domain at the point $(\xi,-\eta)$. Therefore we may deform the path of integration $\partial D^{*}$ in (8.2) back towards the real domain on the far side of the reflecting surface $y=0$.

There are two different cases which can occur. The reason for these is discussed in $\$ 10$.

The first case is that in which the reflected fundamental solution $S^{*}$ exists and satisfies the requirements of $\S 5$ throughout the whole triangular "wedge" bounded by $r^{*}=0$ and $y=0$. In that case the cycle $\partial D^{*}$ may be deformed, by a process which is essentially the reverse of that of $\S 7$, all the way back to form a torus about the image point $(\xi,-\eta, \tau)$. Then by taking the residue as the torus shrinks to a point, one obtains a reflection formula involving data just at the image point $(\xi,-\eta, \tau)$. For example if (3.1) is the heat equation or, more generally, if the coefficients in (3.1) are even functions of $y$, that is precisely what happens. The reflection law obtained in that case is simply

$$
u(\xi, \eta, \tau)=-u(\xi,-\eta, \tau)
$$

The second case is that in which the reflected fundamental solution $S^{*}$ exists and satisfies the requirements of $\S 5$ only in the two triangular "wedges" bounded by 


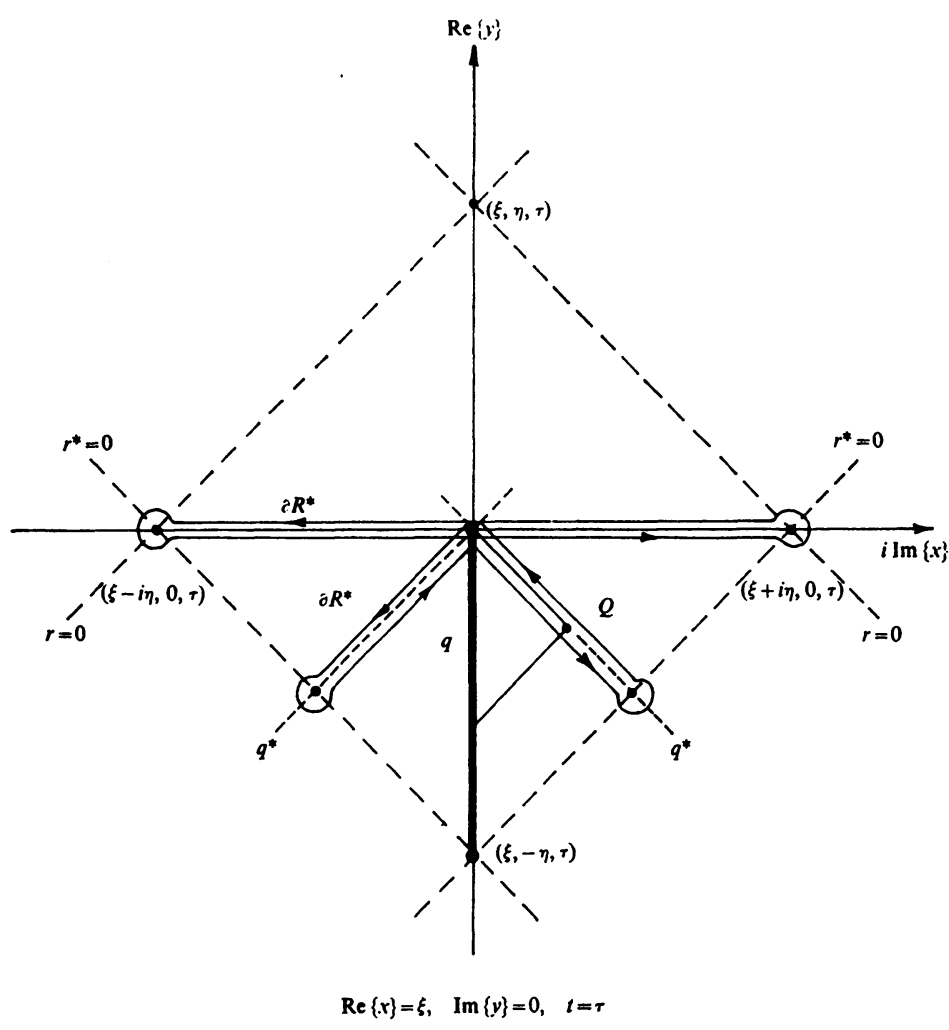

$r^{*}=0, y=0$, and $\operatorname{Re}\{y\}=-|\operatorname{Im}\{x\}|$. Then $\partial D^{*}$ may be deformed back only as far as $\operatorname{Re}\{y\}=-|\operatorname{Im}\{x\}|$. We may thus collapse $\partial D^{*}$ down around the set $q^{*}$

$$
\begin{aligned}
q^{*}:\{(x, y, t) \mid-\eta / 2 \leqq & \operatorname{Re}\{y\} \leqq 0, \operatorname{Im}\{y\} \\
\operatorname{Re}\{x\} & =\xi, \operatorname{Im}\{x\}= \pm \operatorname{Re}\{y\}, t=\tau\} .
\end{aligned}
$$

The resulting residue we denote symbolically by

$$
u(\xi, \eta, \tau)=-\lim _{\partial D^{*} \rightarrow q^{*}} \frac{1}{(2 \pi i)^{2}} \int_{\partial D^{*}} H\left[u, S^{*}\right] .
$$

Equation (8.4) represents $u(\xi, \eta, \tau)$ in terms of Cauchy data along $q^{*}$. This Cauchy data can, in turn, be expressed in terms of data in the real domain along $q$

$$
q:\{(x, y, t) \mid-\eta \leqq y \leqq 0, x=\xi, t=\tau\}
$$

by solving auxiliary Cauchy problems. Indeed the domain of dependence associated with a point $Q$ on $q^{*}$ is a triangle which cuts a segment out of $q$. Let $S$ be the fundamental solution with parameter point at $Q$. Then from (7.2) we have

$$
u(Q)=\frac{1}{(2 \pi i)^{2}} \int_{\partial D} H[u, S] .
$$


We denote symbolically by

$$
u(Q)=\lim _{\partial D \rightarrow q} \frac{1}{(2 \pi i)^{2}} \int_{\partial D} H[u, S]
$$

the residue obtained from (8.5) when $\partial D$ is shrunk down around the relevant segment of $q$. Combining (8.4) and (8.6) the final reflection formula is the iterated residue

$$
u(\xi, \eta, \tau)=-\lim _{\partial D^{*} \rightarrow q^{*}} \lim _{\partial D \rightarrow q} \frac{1}{(2 \pi i)^{4}} \int_{\partial D^{*}} \int_{\partial D} H\left[H[u, S], S^{*}\right] .
$$

Observe that (8.7) expresses $u(\xi, \eta, \tau)$ solely in terms of data along the onedimensional segment $q$. Moreover, it is clear from our discussion of the Cauchy functional in $\$ 7$ that the segment $q$ in (8.7) can be replaced by any path extending from the image point to the reflecting surface.

The second case described above is the more usual one (cf. $\$ 10)$. The occurrence of the first case is an example of an Huygens' principle for reflection which happens only in certain special situations. Note that even when the first case does occur, the reflection functional may still involve an infinite number of $t$-derivatives of $u$ at the image point because of the essential singularity in the reflected fundamental solution. When $u$ is independent of $t$ equation (8.7) reduces to an explicit representation for Lewy's terminal functional [10].

9. Construction of $S$. It is convenient to make the nonsingular complex linear transformations

$$
\begin{array}{rlrl}
z & =x+i y, & \zeta & =\xi+i \eta, \\
z^{*} & =x-i y, \quad \zeta^{*}=\xi-i \eta,
\end{array}
$$

on the space variables. Then equation (4.1) can be rewritten in the form

$$
\mathscr{M}[v] \equiv v_{z z^{*}}+\alpha v_{z}+\beta v_{z^{*}}+\gamma v+(\delta v)_{t}=0 \equiv M[v]+(\delta v)_{t}
$$

where $\alpha=-(a+i b) / 4, \beta=-(a-i b) / 4, \gamma=\left(c-a_{x}-b_{y}\right) / 4$ and $\delta=d / 4$. For convenience we shall often use the same symbols $S, A, B$, etc. to denote the new functions of $z, z^{*}, t, \zeta, \zeta^{*}$, and $\tau$ obtained from the original ones by means of the linear transformations (9.1). Note that, in terms of the new variables, $r^{2}=(z-\zeta)\left(z^{*}-\zeta^{*}\right)$ and $r^{* 2}=\left(z-\zeta^{*}\right)\left(z^{*}-\zeta\right)$

Upon substituting the form (4.2) of $S$ into (9.2) we find

$$
\mathscr{M}[S]=\mathscr{M}[A] \log \frac{1}{r}-2 \frac{A_{z}+\beta A}{z^{*}-\zeta^{*}}-2 \frac{A_{z^{*}}+\alpha A}{z-\zeta}+\mathscr{M}[B]=0 .
$$

Since $A$ and $B$ are to be regular at $z=\zeta$ and $z^{*}=\zeta^{*}$, and since the multiple-valued singularity of the logarithm cannot be cancelled solely by poles, its coefficient

$$
\mathscr{M}[A] \equiv 0
$$


must vanish. In order to cancel the poles at $z=\zeta$ and $z^{*}=\zeta^{*}$ we impose

$$
\begin{aligned}
{\left[\partial / \partial z+\beta\left(z, \zeta^{*}, t\right)\right] A\left(z, \zeta^{*}, t ; \zeta, \zeta^{*}, \tau\right) } & =0 \\
{\left[\partial / \partial z^{*}+\alpha\left(\zeta, z^{*}, t\right)\right] A\left(\zeta, z^{*}, t ; \zeta, \zeta^{*}, \tau\right) } & =0 .
\end{aligned}
$$

If we can find $A$ satisfying (9.4) and (9.5), then $\mathscr{M}[S]=0$ can be achieved by choosing $B$ to be any solution of the inhomogeneous equation

$$
\mathscr{M}[B]=2 \frac{A_{z}+\beta A}{z^{*}-\zeta^{*}}+2 \frac{A_{z^{*}}+\alpha A}{z-\zeta} \equiv f\left(z, z^{*}, t ; \zeta, \zeta^{*}, \tau\right) .
$$

First we expand $A$ in a series

$$
A\left(z, z^{*}, t ; \zeta, \zeta^{*}, \tau\right) \equiv \sum_{j=0}^{\infty} A_{j}\left(z, z^{*}, t ; \zeta, \zeta^{*}\right) j !(t-\tau)^{-j-1}
$$

with coefficients $A_{j}$ that are regular in $t$. The requirement $2^{\circ}$ of $\S 4$ imposes

$$
\begin{aligned}
& A_{0}\left(\zeta, \zeta^{*}, t ; \zeta, \zeta^{*}\right) \equiv i, \\
& A_{j}\left(\zeta, \zeta^{*}, t ; \zeta, \zeta^{*}\right) \equiv 0, \quad j=1,2, \ldots .
\end{aligned}
$$

In order that $(9.5)$ be satisfied it suffices to impose the same condition

$$
\begin{aligned}
{\left[\partial / \partial z+\beta\left(z, \zeta^{*}, t\right)\right] A_{\jmath}\left(z, \zeta^{*}, t ; \zeta, \zeta^{*}\right) } & =0, \\
{\left[\partial / \partial z^{*}+\alpha\left(\zeta, z^{*}, t\right)\right] A_{\jmath}\left(\zeta, z^{*}, t ; \zeta, \zeta^{*}\right) } & =0,
\end{aligned}
$$

for $j=0,1,2, \ldots$ on the coefficients of $A$. Equations (9.9) constitute a system of ordinary differential equations in which the variable $i$ occurs only as a parameter. Taking note of the initial conditions (9.8), we solve them to obtain

$$
\begin{aligned}
& A_{0}\left(z, \zeta^{*}, t ; \zeta, \zeta^{*}\right)=i \exp \left\{-\int_{\zeta}^{z} \beta\left(\sigma, \zeta^{*}, t\right) d \sigma\right\} \\
& A_{0}\left(\zeta, z^{*}, t ; \zeta, \zeta^{*}\right)=i \exp \left\{-\int_{\zeta^{*}}^{z^{*}} \alpha(\zeta, \sigma, t) d \sigma\right\} \\
& A_{j}\left(z, \zeta^{*}, t ; \zeta, \zeta^{*}\right) \equiv 0, \quad j=1,2, \ldots, \\
& A_{j}\left(\zeta, z^{*}, t ; \zeta, \zeta^{*}\right) \equiv 0, \quad j=1,2, \ldots
\end{aligned}
$$

Next we substitute the series (9.7) into (9.4) and obtain

$$
\mathscr{M}[A]=\mathscr{M}\left[A_{0}\right](t-\tau)^{-1}+\sum_{j=1}^{\infty}\left\{\mathscr{M}\left[A_{j}\right]-\delta A_{j-1}\right\} j !(t-\tau)^{-j-1}=0 .
$$

The various powers of $(t-\tau)^{-1}$ must vanish, giving us

$$
\mathscr{M}\left[A_{0}\right]=0, \mathscr{M}\left[A_{j}\right]=\delta A_{j-1}, \quad j=1,2, \ldots
$$

Equations (9.11), together with (9.10), form an infinite recursive sequence of analytic characteristic initial value problems. Observe that the initial values $(9.10)$ 
are prescribed along the complex characteristic "wedge" $r=0$ which, in the real domain, appears as the line $x=\xi, y=\eta$. When the coefficients in (3.1) are independent of $t$, then so are the $A_{j}$, and (9.11), (9.10) collapse to a system of elliptic complex characteristic initial value problems in which $\mathscr{M}$ is replaced by the elliptic operator $M$.

Finally we expand $B$ in a series

$$
B\left(z, z^{*}, t ; \xi, \xi^{*}, \tau\right) \equiv \sum_{j=0}^{\infty} B_{j}\left(z, z^{*}, t ; \zeta, \zeta^{*}\right) j !(t-\tau)^{-j-1}
$$

similar to (9.7). Substituting (9.12) into (9.6) and equating coefficients we obtain

$$
\begin{aligned}
& \mathscr{M}\left[B_{0}\right]=2\left\{\left(A_{0_{z}}+\beta A_{0}\right) /\left(z^{*}-\zeta^{*}\right)+\left(A_{0_{z^{*}}}+\alpha A_{0}\right) /(z-\zeta)\right\}, \\
& \mathscr{M}\left[B_{j}\right]=\delta B_{j-1}+2\left\{\left(A_{j_{z}}+\beta A_{j}\right) /\left(z^{*}-\zeta^{*}\right)+\left(A_{j_{*}}+\alpha A_{j}\right) /(z-\zeta)\right\},
\end{aligned}
$$

for $j=1,2, \ldots$ Observe that by construction the inhomogeneous terms in (9.13) are regular at $r=0$. To obtain unique solutions $B_{j}$ to $(9.13)$ some initial conditions must be imposed. One might, for example, require that the $B_{j}$ vanish along $r=0$.

As a specific example of the procedure described above, consider the heat equation (1.2). The adjoint equation is

$$
\mathscr{M}[v]=v_{x x}+v_{y y}+v_{t}=0 .
$$

In that case we find

$$
A_{j}=i(z-\zeta)^{j}\left(z^{*}-\zeta^{*}\right)^{j} /\left[(j !)^{2} 4^{j}\right], \quad j=0,1,2, \ldots
$$

and the convenient choice of zero initial conditions for the $B_{j}$ yields

$$
B_{0}=0, \quad B_{j}=i \frac{\gamma_{j}(z-\zeta)^{j}\left(z^{*}-\zeta^{*}\right)^{j}}{(j !)^{2} 4^{j-1}}, \quad j=1,2, \ldots
$$

where

$$
\gamma_{j}=1+1 / 2+1 / 3+\cdots+1 / j
$$

Hence for the heat equation our fundamental solution is

$$
S(x, y, t ; \xi, \eta, \tau)=\frac{i}{(t-\tau)} \exp \left\{\frac{r^{2}}{4(t-\tau)}\right\} \log \frac{1}{r}+\frac{4 i}{(t-\tau)} \sum_{j=1}^{\infty} \frac{\gamma_{j}}{j !}\left\{\frac{r^{2}}{4(t-\tau)}\right\}^{j}
$$

which is the same as (1.3).

Note that in the above example (9.15) the infinite series expansions (9.7) and (9.12) for $A$ and $B$ converge because of the factors $(j !)^{2}$ that appear in the denominators of the expressions for $A_{j}$ and $B_{j}$. Such factors will also occur in the general case since the solutions of (9.11) or (9.13) involve the iteration of a Volterra operator which involves essentially two integrations.

10. Construction of $S^{*}$. The method for constructing the reflected fundamental solution $S^{*}$ is similar to that for $S$. 
When the form (5.1) of $S^{*}$ is substituted into (9.2) we find

$$
\begin{aligned}
\mathscr{M}\left[S^{*}\right]= & \mathscr{M}\left[A^{*}\right] \log 1 / r^{*}-2\left(A_{z}^{*}+\beta A^{*}\right) /\left(z^{*}-\zeta\right) \\
& -2\left(A_{z^{*}}^{*}+\alpha A^{*}\right) /\left(z-\zeta^{*}\right)+\mathscr{M}\left[B^{*}\right]=0 .
\end{aligned}
$$

Thus, as in $\$ 9$, we impose

$$
\begin{gathered}
\mathscr{M}\left[A^{*}\right]=0, \\
{[\partial / \partial z+\beta(z, \zeta, t)] A^{*}\left(z, \zeta, t ; \zeta, \zeta^{*}, \tau\right)=0,} \\
{\left[\partial / \partial z^{*}+\alpha\left(\zeta^{*}, z^{*}, t\right)\right] A^{*}\left(\zeta^{*}, z^{*} ; \zeta, \zeta^{*}, \tau\right)=0,}
\end{gathered}
$$

and determine $B^{*}$ by solving an inhomogeneous equation analogous to (9.6). Since the reflecting surface $y=0$ corresponds to $z=z^{*}$ in the new variables, we must also impose the condition

$$
A^{*}\left(z, z, t ; \zeta, \zeta^{*}, \tau\right)=A\left(z, z, t ; \zeta, \zeta^{*}, \tau\right)
$$

We expand $A^{*}$ in a series

$$
A^{*}=\sum_{j=0}^{\infty} A_{j}^{*} j !(t-\tau)^{-j-1}
$$

of the same type as that for $A$ and, as before, we are lead to the system of equations

$$
\mathscr{M}\left[A_{0}^{*}\right]=0, \quad \mathscr{M}\left[A_{j}^{*}\right]=\delta A_{j-1}^{*}, \quad j=1,2, \ldots
$$

The conditions (10.3) and (10.4) on $A^{*}$ imply corresponding conditions on the coefficients $A_{j}^{*}$. Here the requirement (10.4) imposes

$$
A_{j}^{*}\left(z, z, t ; \zeta, \zeta^{*}\right)=A_{j}\left(z, z, t ; \zeta, \zeta^{*}\right)
$$

and these relations replace (9.8) as the set of initial conditions for the system of ordinary differential equations (10.3). Integrating (10.3) we obtain, for $j=0,1,2, \ldots$,

$$
A_{j}^{*}\left(z, \zeta, t ; \zeta, \zeta^{*}\right)=A_{j}\left(\zeta, \zeta, t ; \zeta, \zeta^{*}\right) \exp \left\{-\int_{\zeta}^{z} \beta(\sigma, \zeta, t) d \sigma\right\}
$$

$$
A_{j}^{*}\left(\zeta^{*}, z^{*}, t ; \zeta, \zeta^{*}\right)=A_{j}\left(\zeta^{*}, \zeta^{*}, t ; \zeta, \zeta^{*}\right) \exp \left\{-\int_{\zeta^{*}}^{z^{*}} \alpha\left(\zeta^{*}, \sigma, t\right) d \sigma\right\}
$$

Thus the $A_{j}^{*}$ are to be found as solutions of (10.6) which satisfy not only the initial conditions (10.7) along the analytic extension $z=z^{*}$ of the reflecting surface but also the initial conditions (10.8) along the reflected characteristic $r^{*}=0$. Once the $A_{j}^{*}$ have been found, the coefficients $B_{j}^{*}$ in the expansion

$$
B^{*}=\sum_{j=0}^{\infty} B_{j}^{*} j !(t-\tau)^{-j-1}
$$

are to be determined so as to make $\mathscr{M}\left[S^{*}\right]=0$ by solving inhomogeneous equations similar to (9.13). 
It is instructive to interpret (10.6), (10.7), (10.8) in the domain of $\operatorname{Im}\{y\}=$ $\operatorname{Re}\{x-\xi\}=0$. For convenience we set $\nu=\operatorname{Re}\{y\}, \mu=\operatorname{Im}\{x\}$. Then the characteristic $r^{*}=0$ becomes

$$
\mu^{2}=(\nu+\eta)^{2} .
$$

Thus (10.8) assigns data along the "wedge" (10.10), and (10.7) assigns data along the strip

$$
\nu=0, \quad-\eta \leqq \mu \leqq \eta,
$$

cut out by that wedge. Hence, in this $\mu, \nu, t$-space, we are dealing with a sequence of Goursat-like problems. In fact, using the Cauchy-Riemann equations, we can replace the $v_{x x}+v_{y y}$ term in (4.1) by $v_{v v}-v_{\mu \mu}$. Then (4.1) assumes the form

$$
v_{v \nu}-v_{\mu \mu}+\{\text { lower order terms }\}=0
$$

of an equation with the wave operator as its principal part. When the coefficients in (3.1) are independent of $t$, so are the $A_{j}$ and hence also the $A_{j}^{*}$. Therefore, in that case, the derivative with respect to $t$ may be deleted from (10.12) and then (10.6), (10.7), (10.8) collapse precisely to a sequence of hyperbolic Goursat problems.

The special case mentioned above shows that, in general, we can expect the reflected fundamental solution $S^{*}$ to exist only in the two wedges bounded by (10.10), (10.11), and the central characteristic

$$
\mu^{2}=\nu^{2} \text {. }
$$

Indeed, in order to construct $S^{*}$ we must have both (10.2) and (10.3) satisfied. But the Goursat problems for (10.12) only allow us to assign the data (10.8) along the portion of the characteristic (10.10) between its intersection with (10.11) and (10.13). If we try to impose (10.8) farther along (10.10), we are led to a problem with overdetermined data that may not be solvable. If, on the other hand, we impose (10.2) in the entire region between (10.10) and (10.13) the conditions (10.3) may not be fulfilled and it may not be possible to solve the inhomogeneous equations for $B^{*}$. Thus past the intersection of (10.10) and (10.13) $A^{*}$ may be multiplevalued since it has two determinations obtained by solving characteristic initial value problems: one comes from prescribing data along the central characteristic (10.13) and the other comes from prescribing the data (10.8) along one half of (10.10) past (10.13) together with data prescribed along the corresponding half of (10.11).

\section{BIBLIOGRAPHY}

1. R. J. Duffin, Continuation of biharmonic functions by reflection, Duke Math. J. 22 (1955), 313-324.

2. V. Filippenko, On the reflection of harmonic functions and of solutions of the wave equation, Pacific J. Math. 14 (1964), 883-893.

3. P. R. Garabedian, Partial differential equations with more than two independent variables in the complex domain, J. Math. Mech. 9 (1960), 241-271. 
4. P. R. Garabedian, Analyticity and reflection for plane elliptic systems, Comm. Pure Appl. Math. 14 (1961), 315-322.

5. —-, Partial differential equations, Wiley, New York, 1964.

6. J. Hadamard, Lectures on Cauchy's problem in linear partial differential equations, Dover, New York, 1952.

7. C. D. Hill, Parabolic equations in one space variable and the non-characteristic Cauchy problem, Comm. Pure Appl. Math. 20 (1967), 619-633.

8. A. Huber, The reflection principle for polyharmonic functions, Pacific J. Math. 5 (1955), 433-439.

9. F. John, Continuation and reflection of solutions of partial differential equations, Bull. Amer. Math. Soc. 63 (1957), 327-344.

10. H. Lewy, On the reflection laws of second order differential equations in two independent variables, Bull. Amer. Math. Soc. 65 (1959), 37-58.

11. - On the extension of harmonic functions in three variables, J. Math. Mech. 14 (1965), 925-927.

12. H. Poritsky, Applications of analytic functions to two-dimensional biharmonic analysis, Trans. Amer. Math. Soc. 59 (1946), 258.

13. A. Sommerfeld, Partial differential equations in physics, Academic Press, New York, 1949.

\section{ROCKEFELLER UNIVERSITY,} New York, New YoRK 